\title{
WAP four-disulfide core domain protein 2 mediates the proliferation of human ovarian cancer cells through the regulation of growth- and apoptosis-associated genes
}

\author{
YAO CHEN ${ }^{1}$, XIA MU $^{2}$, SUIHAI WANG ${ }^{1}$, LIANG ZHAO ${ }^{3}$, YINGSONG WU ${ }^{1}$, JILIANG LI ${ }^{1,4}$ and MING LI ${ }^{1}$ \\ ${ }^{1}$ School of Biotechnology, Southern Medical University, Guangzhou 510515; ${ }^{2}$ Guizhou Provincial People's Hospital, \\ Guiyang, Guizhou 550002; ${ }^{3}$ Department of Pathology, School of Basic Medical Sciences, Southern Medical University, \\ Guangzhou 510515, P.R. China; ${ }^{4}$ Cancer Research UK (CRUK) Molecular Oncology Laboratories, Department of Oncology, \\ Weatherall Institute of Molecular Medicine, University of Oxford, Oxford OX3 9DS, UK
}

Received July 23, 2012; Accepted September 28, 2012

DOI: 10.3892/or.2012.2114

\begin{abstract}
The WAP four-disulfide core domain protein 2 (WFDC2) is frequently overexpressed in epithelial ovarian cancer cells and has been proposed as a potential biomarker. The biological function of WFDC2 in tumor progression remains unclear. In this study, the stable expression of short hairpin RNA (shRNA) against WFDC2 in the human ovarian SKOV3 cell line was established. Cell proliferation in vitro was determined by MTT assay. Cell cycle and apoptosis were analyzed by FACS. The expression of genes related to cell proliferation and survival was detected by real-time RT-PCR and western blotting. In vivo tumor growth assay was performed by establishing WFDC2-knockdown xenografts in nude mice and monitoring tumor growth. The expression of WFDC2, Ki67 and activated caspase-3 was analyzed by immunohistochemistry in order to determine the role of WFDC2 in proliferation and apoptosis. Our results revealed that the silencing of WFDC2 abolished ovarian cancer cell proliferation, suppressing tumor formation and growth in ovarian cancer cells both in vitro and in vivo. The knockdown of WFDC2 induced upregulation of Fasl and the downregulation of cyclin D1 activated caspase-3 and Ki67. These results indicate that WFDC2 plays a crucial role in tumor formation and growth in ovarian cancer cells. WFDC2 may be a potential therapeutic target for epithelial ovarian cancer.
\end{abstract}

Correspondence to: Dr Ming Li, School of Biotechnology, Southern Medical University, Guangzhou 510515, P.R. China E-mail: mingli2006_2006@126.com

Dr Jiliang Li, Cancer Research UK (CRUK) Molecular Oncology Laboratories, Department of Oncology, Weatherall Institute of Molecular Medicine, University of Oxford, Oxford OX3 9DS, UK E-mail: ji-liang.li@imm.ox.ac.uk

Key words: WAP four-disulfide core domain protein 2, ovarian cancer, cell proliferation, apoptosis

\section{Introduction}

Ovarian cancer is one of the most common cancers among women, and it is the leading cause of mortality among gynecological malignancies worldwide. WAP four-disulfide core domain protein 2 (WFDC2) was first identified in the epithelium of the distal epididymis and was originally predicted as a protease inhibitor involved in sperm maturation. Recent interest in WFDC2 has been generated by the consistent demonstration of its overexpression in ovarian carcinomas in comparison to normal ovarian tissue (1-3). This gene, also known as human epididymis 4 (HE4), has been consistently identified as being upregulated in ovarian carcinoma by gene expression profiling studies and has been proposed as a putative serum biomarker for malignant ovarian masses $(1,4-6)$.

The WFDC2 gene is located on human chromosome 20q1213.1. The amplification of $20 q 12-13$ has been demonstrated specifically in breast and ovarian carcinomas $(2,7)$. Fourteen genes which encode proteins with a WAP-type four-disulfide core (WFDC) domain have been identified on this region. Two of these genes, SLPI and p13, encoded as antileukoproteinase 1 and elafin, which are co-expressed with WFDC2 play a role in the development or progression of various types of carcinomas $(8,9)$. This led us to investigate whether WFDC2, a member of the WAP cluster, overexpressed in ovarian cancer, may also be involved with this disease.

The most studied WAP proteins, p13 and SLPI, both identified as serine-proteinase inhibitors, are reported to be associated with aggressive, high-risk, or metastatic cancer originating from various organs $(10,11)$. The expression of SLPI was positively correlated with the increased expression of the cell cycle progression factor, cyclin D1, and its causal role in the promotion of malignant behavior was also demonstrated in lung carcinomous cells stably transfected with human SLPI-expression constructs $(8,12-14)$. Elafin was reported to be overexpressed in late-stage, high-grade serous ovarian carcinomas and to correlate with poor overall survival (15). In breast cancer cells and tissue, elafin was found to protect cyclin E from proteolysis with elastase, thus regulating cell cycle progression (16). Both SLPI and elafin may regulate 
$\mathrm{NF}-\kappa \mathrm{B}$ activity induced by inflammatory cytokines; their tumor proliferation promotion and spread may be involved in the regulation of $\mathrm{NF}-\kappa \mathrm{B}$ activity $(17,18)$.

Although, no antiproteinase-inhibitory activity has been assigned to WFDC2 and the biological role of WFDC2 remains unclear, on the basis of the similarity to SLPI and p13, we hypothesized that WFDC2 has a pro-cancer function in ovarian cancer by regulating tumor proliferation and facilitating tumor spread through a tissue remodeling method. To explore the functional role of WFDC2 in ovarian cancer, we performed the transfection of SKOV3 cells with short hairpin RNA (shRNA) against WFDC2 and investigated the effect of WFDC2 silencing on cell proliferation, tumorigenesis, cell cycle and apoptosis.

\section{Materials and methods}

Materials and animals. Reagents, enzymes and laboratory supplies were purchased from the following vendors: restriction enzymes from Takara; MTT from Sigma (Shanghai, China); G418 (neomycin) and TRIzol reagent from Invitrogen Life Technologies; cell culture media, antibiotics/serum and glutamine from Gibco-BRL. All other molecular reagents and solvents, when not listed, were purchased from Sigma. All other reagents were of molecular or imaging grade. 6- to 8-weekold female BALB/c-nu mice used in these experiments were purchased from the Experimental Animal Center of Southern Medical University (P.R. China) and were maintained under standard pathogen-free conditions.

Gene silencing. SKOV3, human ovarian carcinoma cells, were selected for these experiments. Four different WFDC2-specific target sequences were designed and constructed using the WFDC2 reference sequence (Gene Bank Accession no. NM_0006103.3). The target sequences of WFDC2-209, WFDC2-216, WFDC2-308 and WFDC2-318 are homologous to 5'-GCTCTCTGCCCAATGATAAGG3', 5'GCCCAAT GATAAGGAGGGTTC3'，5'GTGTCCTGGCCAGATG AAATG-3' and 5'-GATGAAATGCTGCCGCAATGG-3' of the WFDC2-specific mRNA, respectively, with an invalid RNAi sequence of 5'-GTTCTCCGAACGTGTCACGT-3' as the negative control. The shRNAs were chemically synthesized and the shRNA expression vector (WFDC2-pGPU6/ Neo) was constructed by Shanghai Genepharma Co., Ltd. The correct insertion of the specific shRNA was further confirmed by sequencing. The SKOV3 cell line was transfected with WFDC2-specific shRNA-encoding expression vectors using Lipofectamine 2000 (Invitrogen Life Technologies). For stable silencing of WFDC2, the transfected SKOV3 cell line was selected by G418. The G418-resistant colonies were selected, expanded and analyzed separately.

In vitro cell proliferation assay. In vitro proliferation assay was performed using the MTT assay following the manufacturer's instructions (Sigma). Cells were incubated at 500 cells/well in 96-well plates in triplicates and cultured in complete DMEM/F12 medium (10\% FBS) to observe the growth rate. For 6 days, the absorbance of the cells was measured using the 3-(4,5-dimethylthiazol-2-yl)-2,5-diphenyltetrazolium bromide (MTT) method, and the cell growth curves were calculated.
Colony formation assay. Cells were planted in 24-well plastic plates spread with low-melting point agar $(0.35 \%$ in the upper layer and $0.6 \%$ in the lower layer). Each type of cell was planted in 5 wells, with 100 cells/well. The cells were cultured at $37^{\circ} \mathrm{C}$ with $5 \% \mathrm{CO}_{2}$ and under saturation humidity for 14 days. Cell colonies consisting of more than 50 cells were counted under an inverted microscope. The colony formation rate was calculated as follows.

Colony efficiency $=\frac{\text { average number of colonies of } 5 \text { wells }}{\text { number of plated cells }} \times 100 \%$

RNA extraction and real-time RT-PCR. Total RNA was isolated following the manufacturer's instructions (PrimeScript 1st Strand cDNA Synthesis kit, Takara). Reverse transcription was also performed following the manufacturer's instructions in a total volume of $20 \mu \mathrm{l}$ using an Oligo(dT) primer and $1 \mu \mathrm{g}$ of total RNA. Each primer set was designed to flank an intron to prevent the amplification of genomic DNA, using Primer Express 3.0. The forward and reverse primers, respectively, are shown in Table I. $\beta$-actin was used to evaluate the efficiency and variability of the reverse transcription step. cDNA samples $(0.1 \mu \mathrm{g})$ were amplified using the SYBR-Green PCR Master Mix (Takara) under conditions recommended by the manufacturer: i) pre-incubation at $95^{\circ} \mathrm{C}$ for $30 \mathrm{sec}$; ii) $40 \mathrm{PCR}$ cycles of $95^{\circ} \mathrm{C}$ for $5 \mathrm{sec}, 55^{\circ} \mathrm{C}$ for $30 \mathrm{sec}$ and $72^{\circ} \mathrm{C}$ for $34 \mathrm{sec}$. Samples were assayed in duplicate using the ABI Prism 7500 detection system (Perkin Elmer Applied Biosystems). Relative quantification was then calculated by subtracting the average $\mathrm{CT}$ from the corresponding average $\mathrm{CT}$ for $\beta$-actin.

Western blot analysis. Cells were harvested and lysed in ice-cold lysis buffer [10 mM HEPES (pH 7.9), $150 \mathrm{mM}$ $\mathrm{NaCl} ; 1 \mathrm{mM}$ EDTA]. Proteins were fractionated on SDS-polyacrylamide gels and transferred to a nitrocellulose membrane. Immunoblotting was performed with goat polyclonal anti-human WFDC2 (Santa Cruz Biotechnology, Inc.), goat polyclonal anti-human P21, goat polyclonal antihuman $\mathrm{Rb}$, goat polyclonal anti-human $\mathrm{Bcl} 2$, goat polyclonal anti-human Bax, goat polyclonal anti-human cyclin D1, goat polyclonal anti-human CDK4 and goat polyclonal anti-human activated caspase-3 antibodies (Cell Signaling Technology, Inc.). Anti-goat secondary antibody conjugated to horseradish peroxidase (Santa Cruz Biotechnology, Inc.) was used to visualize the stained bands with an enhanced chemiluminescence (ECL) visualization kit (Amersham).

Flow cytometry. Cells were trypsinized and washed with phosphate-buffered saline (PBS). The cells were incubated in $4 \%$ paraformaldehyde and fixed at $4^{\circ} \mathrm{C}$ for $30 \mathrm{~min}$. After washing 3 times, cells were digested with $1 \%$ RNase at $37^{\circ} \mathrm{C}$ for $30 \mathrm{~min}$ and stained with $50 \mu \mathrm{g} / \mathrm{ml}$ propidium iodide (PI; Sigma, Shanghai, China) for $1 \mathrm{~h}$ at $4^{\circ} \mathrm{C}$ for cell apoptosis and cycling assay. The results were analyzed using WinMDI software and 10,000 events for each sample were collected. Cell suspensions were incubated with antibodies to Fas and Fasl ( $1 \mu \mathrm{g} / 1 \times 10^{6}$ cells) for $30 \mathrm{~min}$ at $37^{\circ} \mathrm{C}$, washed 3 times with PBS and incubated with PE- and FITC-labeled secondary antibodies for $30 \mathrm{~min}$ at $37^{\circ} \mathrm{C}$. The expression of Fas and Fasl/10,000 cells was determined by flow cytometry. 
Table I. Primers for real-time RT-PCR assay.

\begin{tabular}{|c|c|c|}
\hline Primers & $5^{\prime} \rightarrow 3^{\prime}$ & $\begin{array}{c}\text { Annealing } \\
\operatorname{Tmp}\left({ }^{\circ} \mathrm{C}\right)\end{array}$ \\
\hline
\end{tabular}

Apaf-1 F: ACAATGCTCTACTACATGAAGGATATAAAGA 58 R: CACTGGAAGAAGAGACAACAGGAA

BCL2L11 F: ATCCCCGCTTTTCATCTTTA R: AGGACTTGGGGTTTGTGTTG

Bax F: GCTGTTGGGCTGGATCCAAG R: TCAGCCCATCTTCTTCCAGA

BCL-X F: CATGGCAGCAGTAAAGCAAG R: TAGAGTTCCACAAAAGTATC

Cyclin D1F: CGCCCCACCCCTCCAG R: CCGCCCAGACCCTCAGACT

Cyclin E1 F: TCCAGGAAGAGGAAGGCAAAC R: CCTGTCGATTTTGGCCATTT

Casp3 F: GAGTGCTCGCAGCTCATACCT R: CCTCACGGCCTGGGATTT

Casp7 F: GAAGCCCTCTGCTCCATCC R: GGCAACTCTGTCATTCACCCT

Casp9 F: GGGTCGCTAATGCTGTTTCG R: TGCAAGATAAGGCAGGGTGAG

gadd45a F: TCAGCGCACGATCACTGTC R: CCAGCAGGCACAACACCAC

RB F: ACTCCGTTTTCATGCAGAGACTAA R: GAGGAATGTGAGGTATTGGTGACA

TP53BP2 F: GGATGGGTATGATGGGACAG R: GGGCCAAATATTCAGAAGC

P73 F: GGATTCCAGCATGGACGTCTT R: GCGCGGCTGCTCATCT

P21 F: TGGACCTGTCACTGTCTTGTACC R: CTTCCTGTGGGCGGATTAG

P53 F: TCTGACTGTACCACCATCCACTAC R: CAGGCACAAACACGCAC

P73 F: CATGGAGACGAGGACACGTACTA R: CCATCAGCTCCAGGCTCTCT

KI67 F: GAGTGCCTGCTCAGTGTTGG R: GCCACACTGTGTCGTCGTTT

AKT1 F: TCCGAGCTGTTCTTCCACC R: CTTGATCCCCTCCTTGCAC

CD44 F: TTTGCATTGCAGTCAACAGTC R: GTTACACCCCAATCTTCATGTCCAC

Fas F: ACACTGTGACCCTTGCACCAA R: AGCCACCCCAAGTTAGATCTG

FasL F: GGCCTGTGTCTCCTTGTGAT R: TGCCAGCTCCTTCTGTAGGT

$\beta$-actin $F$ : TTGTTACAGGAAGTCGCTTGCC R: ATGCTATCACCT CCCCTGTGTG

Xenograft formation. The animal study was divided into two parts. Firstly, 10 mice were divided into 2 groups and, SKOV3-209 and SKOV3-NA cells $\left(1 \times 10^{6}\right)$ were injected into the flank of 6- to 8-week-old female BALB/c-nu mice, respectively. The SKOV3-209 and SKOV3-NA xenografts were excised at 6 weeks. The tumor volume (V) was calculated as $\mathrm{V}=\mathrm{W} \times \mathrm{W} \times \mathrm{L} \times \mathrm{0.52}$. Secondly, the tumor volume (V) was measured at different time points by using a caliper. The tumor volume (V) was calculated as $\mathrm{V}=\mathrm{W} \times \mathrm{W} \times \mathrm{L} \times 0.52$ and the xenografts were harvested for subsequent analysis by being fixed in buffered neutral formalin when reaching $200 \mathrm{~mm}^{3}$.

Immunohistochemistry. Immunohistochemistry was performed using 8-to $10-\mu \mathrm{m}$ serial sections of fixed tissue placed onto positively charged glass slides using a single-staining procedure. The protocols used with each antibody are provided below.

Anti-WFDC2 monoclonal, anti-Ki67 rabbit polyclonal and anti-activated caspase-3 rabbit polyclonal antibodies were applied to the slides at a dilution of 1:2,000 and incubated overnight at $4^{\circ} \mathrm{C}$. The slides were then stained using the avidinbiotin method, as described above. 3,3'-Diaminobenzidine chromogen was used to develop a brown color for $5 \mathrm{~min}$. Slides were lightly counterstained with hematoxylin. Tumor cells were considered positive for the antigen when there was brown colored staining. The intensity of WFDC 2 immunostaining of the xenografts was scored as negative (0), weak (1), medium (2) and strong (3). The extent of staining, defined as the percentage of positive-stained cells, was scored as $1(\leq 10 \%)$, $2(11-50 \%), 3(51-75 \%)$ and $4(>75 \%)$. An overall expression score, ranging from 0 to 12 , was calculated by multiplying the score of the staining intensity and the score of the extent of staining. The final staining score was presented as the negative (overall score of 0 ), $1^{+}$(overall score of $0-3$ ), $2^{+}$(overall score of 4) or $3^{+}$(overall score of $\geq 5$ ).

Ki67 is located in the cell nucleus. At least 500 tumor cell nuclei for each xenograft ( $\mathrm{n}=5)$ of the SKOV3-209 or SKOV3-NA groups were randomly selected and examined. The number of Ki67-positive tumor cell nuclei was counted and the Ki67-positive index was calculated as follows: Ki67 index $=$ (number of apoptotic cells/total cell number) $\mathrm{x} 100 \%$.

Statistical analysis. Microsoft Office Excel 2007 and the statistical software SPSS 13.0 were used in data processing and in analyzing statistical significance with one-way ANOVA. P-values $<0.05$ were considered to indicate a significant difference. Data are expressed as the means \pm SD from at least 3 independent experiments.

\section{Results}

WFDC2 gene silencing. shRNA targeting WFDC2 was used to knockdown the WFDC2 expression in SKOV3 cells. The silencing efficiency was detected by real-time quantitative PCR and western blotting. shRNA reduced the WFDC2 expression in both the SKOV3-209 and -309 sublines (Fig. 1). The shRNA targeting sequence 309 and 209 decreased WFDC 2 mRNA by up to 73.1 and $86.3 \%$, respectively (Fig. 1). The transfection of shRNA 209 induced a significant downregulation of WFDC2 expression in all shRNA clones. Therefore, the stable cell line SKOV3-209 was selected for subsequent analysis. Cells transfected with an invalid RNAi sequence, namely SKOV3-NA, were used as the negative control. 
A

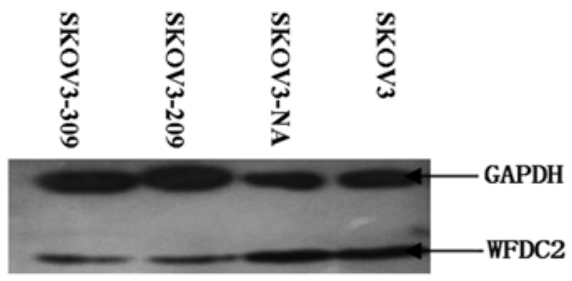

B

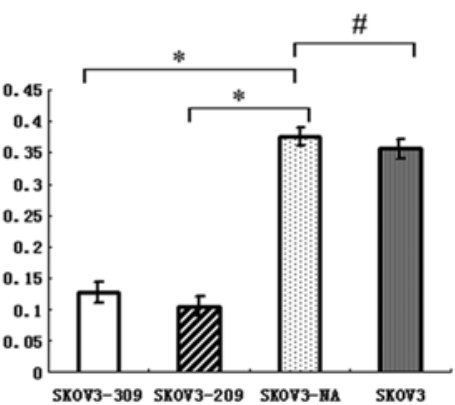

Figure 1. Expression of WFDC2 in WFDC2-silenced clonal lines. (A) Western blot analysis of the expression of WFDC2 and GAPDH. (B) Normalized WFDC2 protein levels in the shRNA-transfected, mock-transfected NA and the control. The relative quantities of WFDC2 protein were determined by densitometry and normalized using GAPDH. ${ }^{*} \mathrm{P}<0.05$ compared to SKOV3-NA; ${ }^{*} \mathrm{P}<0.05$ compared to SKOV3.

A

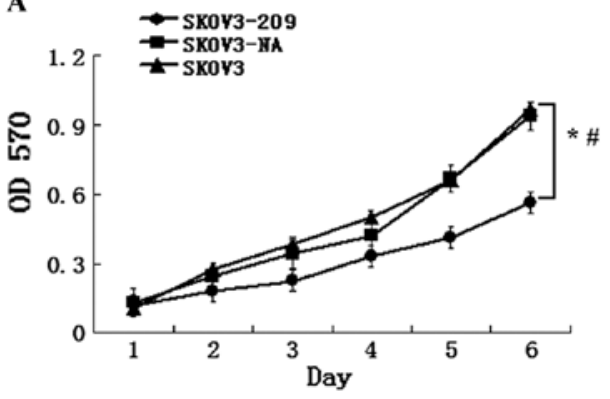

B

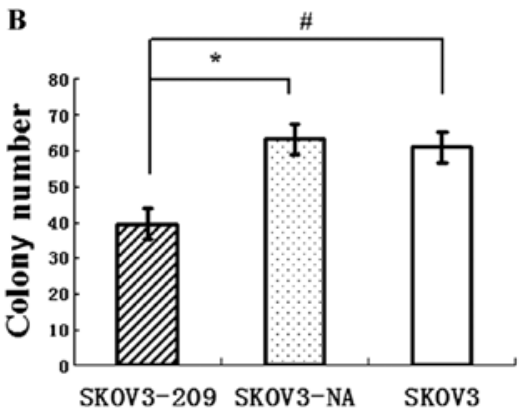

Figure 2. Knockdown of WFDC2 expression inhibits cell growth in vitro. (A) Proliferation curve of SKOV3 cells treated with an empty vector control (SKOV3-NA) and WFDC2 knockdown (SKOV3-209) or PBS (SKOV3) over 6 days measured by MTT assay. "P $<0.05$ compared to SKOV3-NA; ${ }^{\mathrm{P}} \mathrm{P}<0.05$ compared to SKOV3 (B) Histogram plots represent the number of colonies at day 6 in 3 separate experiments performed in triplicate. Data presented in A and B (means \pm SE) are from 3 independent experiments, with each experiment performed in triplicate. ${ }^{*} \mathrm{P}<0.05$ compared to SKOV3-NA; ${ }^{~} \mathrm{P}<0.05$ compared to SKOV3.

Knockdown of WFDC2 expression inhibits cell growth in vitro. MTT assay and colony formation assays were conducted to determine the cell growth. The growth curves indicated that the growth rates did not have marked differences among the SKOV3-209, SKOV3-NA and SKOV3 cells on the first day after transfection (Fig. 2A). However, on each day for the following five days, the growth rate was significantly lower in SKOV3-209 cells compared to that in SKOV3-NA and SKOV3 cells (Fig. 2A). Six days after plating, the cell density observed for the control line was nearly 1.5 -fold higher compared to that of the WFDC2 shRNA-transfected subline. The average colony formation rate in SKOV3-NA was $62.4 \pm 4.08 \%$. After being transfected with shRNA, the average colony formation rate of SKOV3-209 cells was 37.4 $\pm 2.97 \%$, with an inhibition rate of $39.98 \%$. These results indicate a positive correlation between WFDC2 expression and ovarian cancer cell growth (Fig. 2B).

Genes related to cell proliferation and survival were modified by WFDC2 knockdown. To determine the effect of WFDC2 knockdown on SKOV3 cell proliferation and survival, the mRNA levels for a number of genes related to cell proliferation and survival were detected in the shRNA-silenced lines and, for comparison, in the SKOV3-NA cell line by real-time RT-PCR. When normalized to the expression of the control $\beta$-actin gene, which was unaffected by cellular WFDC2 expression, a slight decrease in cyclin D1, cyclin E, p21, caspase-3 and Bcl2 expression was observed at the mRNA level in SKOV3-209 cells (Fig. 3A). However, Fasl was increased approximately 8-fold (Fig. 3A). We also evaluated the expression of these genes at the protein level. According to western blot results, the downregulation of cyclin D1 and activated caspase-3 expression was also observed at the protein level, while no difference in the expression of other cell cycle- and apoptosis-related genes was detected by western blotting (Fig. 3B-D).

Fas/Fasl is a membrane protein related to cell apoptosis. As a primary membrane receptor, we used flow cytometry to assess the expression of Fas/Fasl. The results indicated that the expression of Fasl was upregulated by WFDC2 knockdown while no change was observed in Fas (Fig. 3E). WFDC2 knockdown affected the expression of cyclin D1, caspase-3 and Fasl which are related to the proliferation and apoptosis of tumor cells.

WFDC2 knockdown arrests the cell cycle but has little effect on cell apoptosis. To investigate the potential function of WFDC2 in the proliferation and survival of ovarian cancer cells, the cell cycle and apoptosis of SKOV3 cells were assessed by flow cytometry. The percentage of SKOV3-209 and SKOV3-NA cells in the G0/G1 phase was $79.38 \pm 3.08$ and $63.1 \pm 2.03 \%$, respectively. The percentage of cells in the $S$ phase for these two groups was $18.47 \pm 2.06$ and $39.82 \pm 1.89 \%$, respectively (Fig. 4A and B). These results indicated that a significant percentage of SKOV3 cells was arrested in the G0/G1 phase after treatment with WFDC2 shRNA. The apoptosis rate was also examined by flow cytometry. Although western blot and flow cytometry results demonstrated that activated caspase-3 and Fasl expression was significantly decreased or increased, respectively in the SKOV3-209 cells, the apoptosis 

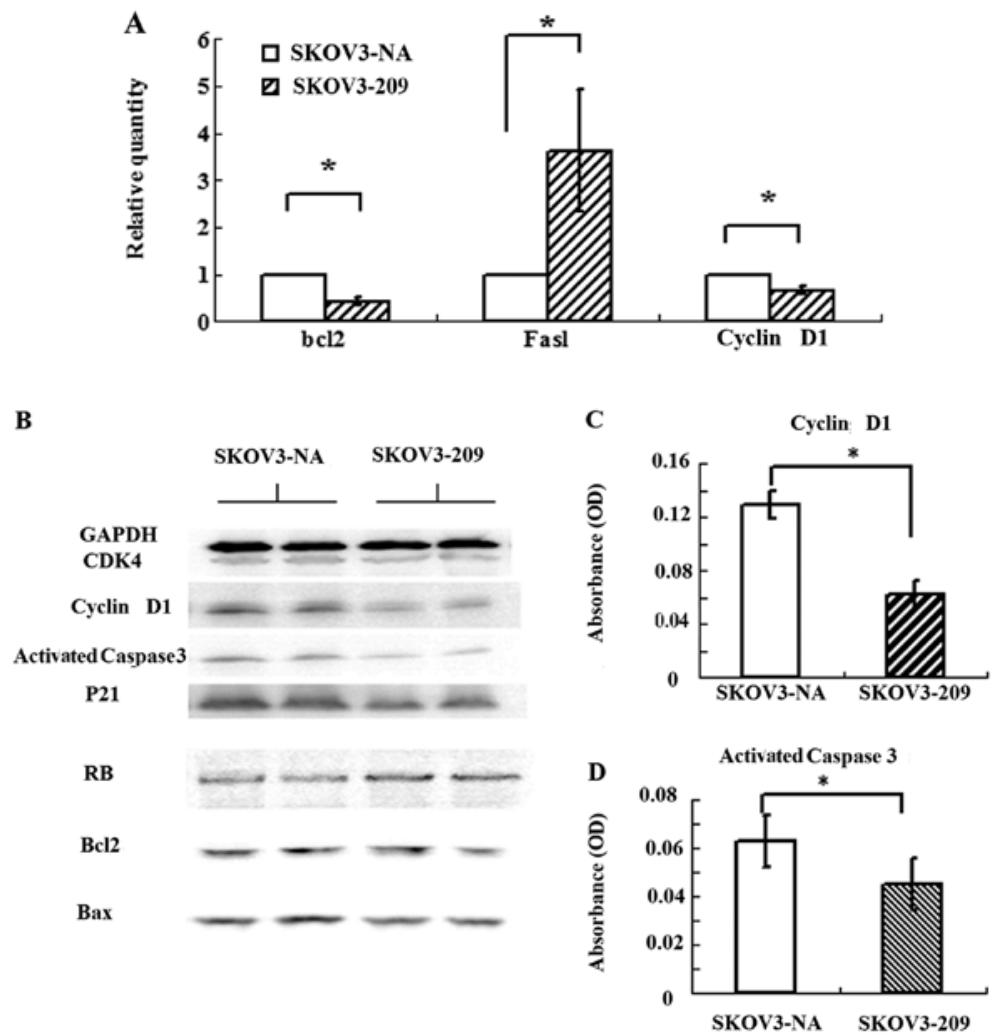

$\mathbf{E}$ SKOV3-NA

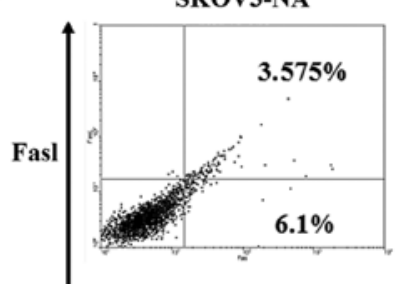

SKOV3-209

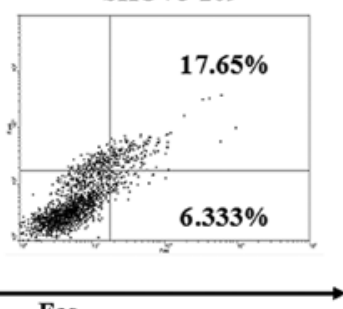

Fas
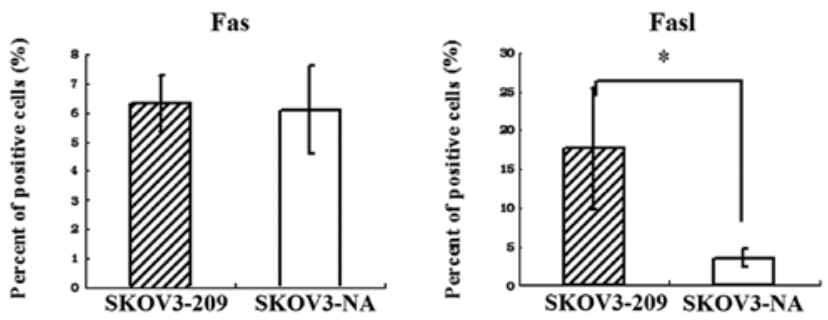

Figure 3. Genes related to cell proliferation and survival are modified by WFDC2 knockdown. (A) Normalized cell cycle- and apoptosis-related gene mRNA levels in the SKOV3-NA and SKOV3-209 cell lines. The relative quantities of the WFDC2 protein were determined by densitometry and normalized using $\beta$-actin. "P $<0.05$ compared to SKOV3-NA. (B) Western blot analysis of the expression of cell cycle- and apoptosis related-genes and GAPDH. (C) Normalized cyclin D1 protein level in the SKOV3-NA and SKOV3-209 cell lines with GAPDH. "P<0.05 compared to SKOV3-NA. (D) Normalized activated caspase-3 protein level in the SKOV3-NA and SKOV3-209 cell lines with GAPDH. "P<0.05 compared to SKOV3-NA. (E) The percentage of Fas/Fasl-positive cells detected by flow cytometry. "P<0.05 compared to SKOV3-NA.

rate displayed no significant change between SKOV3-209 and SKOV3-NA cells in vitro. No difference was observed between the WFDC2 knockdown cells and the negative control cells in vitro (data not shown).

WFDC2 is required for SKOV3 cell tumorigenesis. To investigate the effect of WFDC2 knockdown on tumorigenesis in vivo, an ovarian carcinoma xenograft model was established. The short time course of tumor formation minimized confounding effects related to the possible loss of WFDC2 expression in mice. SKOV3-NA and SKOV3-209 cells were injected into the groin of nude mice. In mice injected with cells containing an inducible clone of shRNA WFDC2, tumor growth was delayed for several days after the induction of shRNA and tumor sizes were statistically different at every measurement time point after Day 18 (Fig. 5A). Loss of WFDC2 expression resulted in a significant delay in tumor growth, where the difference in tumor 

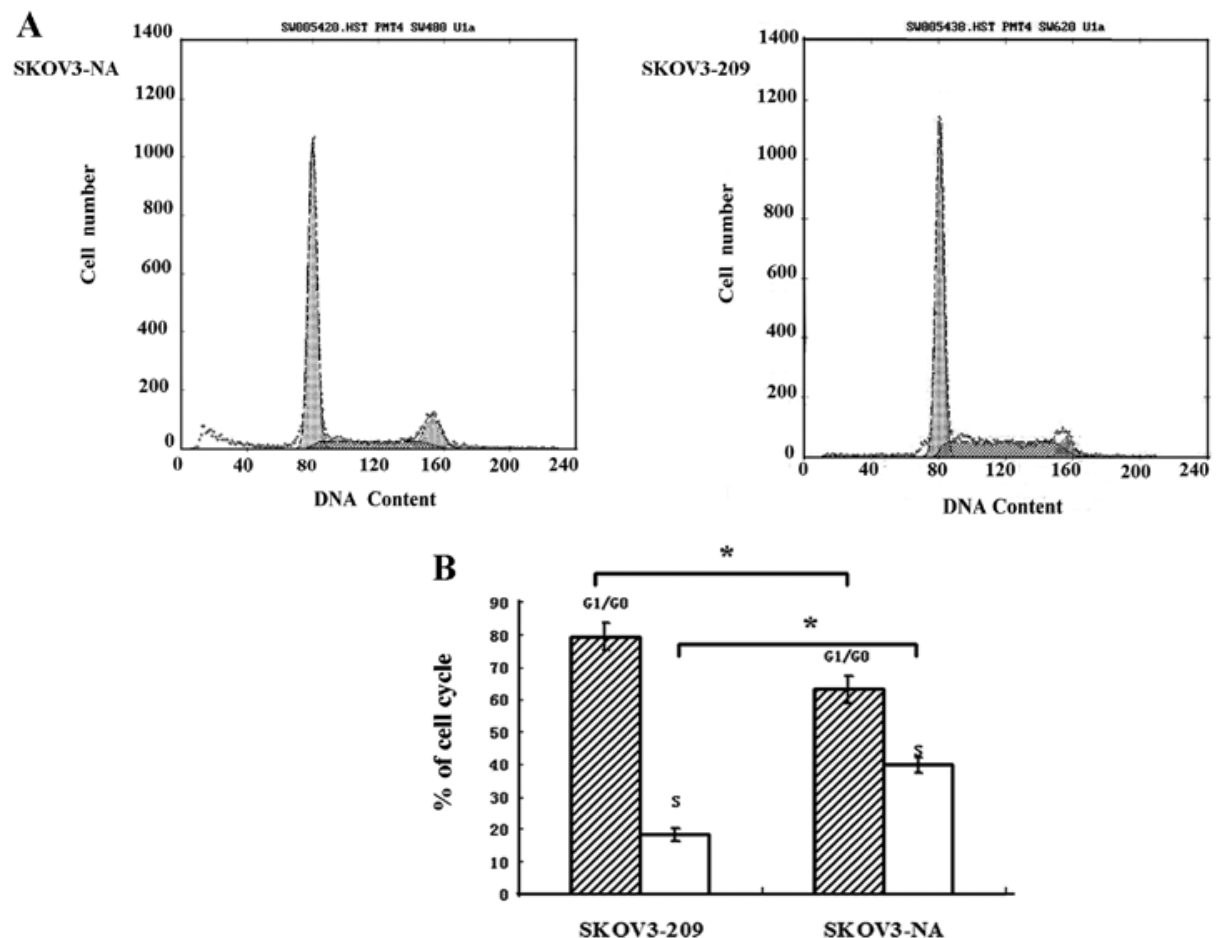

Figure 4. WFDC2 knockdown arrests the cell cycle but has little effect on cell apoptosis. (A) Flow cytometry graphs for SKOV3-NA and SKOV3-209 cell lines. (B) Quantification of the percentages of cells in the G0/G1 and S phases. "P $<0.05$ compared to SKOV3-NA.

volume was statistically significant after 6 weeks (Fig. 5B). To explore the mechanisms of tumor inhibition in vivo the animals were sacrificed when the tumor volume was $\sim 200 \mathrm{~mm}^{3}$. Analysis of WFDC2 protein expression by immunohistochemistry confirmed a reduced cytoplasmic and secreted expression in SKOV3-209 mice after six weeks. The average tumor volume was 8.9-fold larger in the SKOV3-NA group compared to that in the SKOV3-209 group $(\mathrm{P}<0.05)$ (Fig. 5B).

Cell proliferation and apoptosis were also observed in vivo through a tumorigenesis assay. The Ki67 protein is present during all active phases of the cell cycle (G1, S, G2 and mitosis), but is absent from resting cells (G0), making it an excellent marker for determining the cell cycle ratio. The fraction of Ki67-positive tumor cells is often correlated with the clinical course of cancer. Therefore, Ki67 is an important indicator of tumor proliferation in vivo. In our study, with WFDC2 knockdown (Fig. 5C), Ki67 staining demonstrated that nuclear localization of the protein was reduced when WFDC2 was downregulated (Fig. 5C). These results confirm the relationship between the WFDC2 and cell cycle of cancer cells.

In SKOV3-209 xenografts, we noted the presence of activated caspase- 3 in the nucleus of the tumor cells, which was almost absent in the SKOV3-NA controls (Fig. 5D). However, the activated caspase-3-positive cells were in a limited proportion of the total cells. Our results suggest that further studies are required to determine the relationship between apoptosis and WFDC2.

\section{Discussion}

Genomic analysis of ovarian cancer identified amplification of WFDC2 and the whey acidic protein locus in a large proportion of epithelial ovarian cancers $(1,4,6,9,11)$. WFDC2 demonstrates a tumor-restricted, upregulated pattern of expression, making it a potential marker $(3,5,7)$.

WFDC2 contains two WAP structural domains and belongs to the WAP protein family. Previous research indicates that proteins with a WFDC motif usually exhibit the following: A number of WAP proteins have been mapped to human chromosome 20q12-13.1, a region amplified in several types of cancers leading to the proposal that genes located on this region of chromosome 20 play a role in carcinogenesis and tumor progression $(6,9)$; exhibit anti-inflammatory and antimicrobial activity against Gram-negative bacteria and viruses by regulating the $\mathrm{NF}-\kappa \mathrm{B}$ activity $(17-19)$; regulate cell growth $(13,14,20,21)$ and regulate angiogenesis and cell spread by the interference of the leucocyte cell-derived and tumor cell-derived proteinase and cytokines $(8,12,22,23)$. Despite these findings the precise function of WFDC2 remains unknown.

In this study, we constructed a WFDC2 shRNA expression plasmid, which achieved efficient and specific WFDC2 gene silencing in the human ovarian cancer cell line SKOV3. With WFDC2 gene silencing, we studied the function of WFDC2 in cancer cell growth and survival. To examine whether the modulation of WFDC2 expression influences the tumorigenic properties of ovarian cancer cells, we examined SKOV3 cell proliferation and apoptosis in vitro. As demonstrated by both the MTT assay and FACS analysis, loss of WFDC2 expression resulted in a significant delay in tumor growth in vitro and the cell cycle was arrested in the G0/G1 phase. Furthermore, the injection of SKOV3-NA and SKOV3-209 cells into nude mice demonstrated that in vivo silencing of WFDC2 expression in ovarian cancer cells suppressed tumor growth. According to these results, WFDC2 may play an oncogenic role in ovarian cancer progression through promoting cell proliferation. 
A

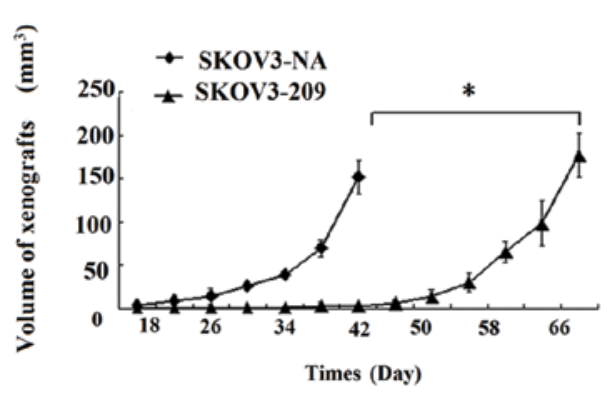

B

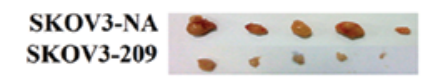

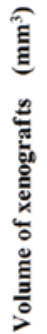

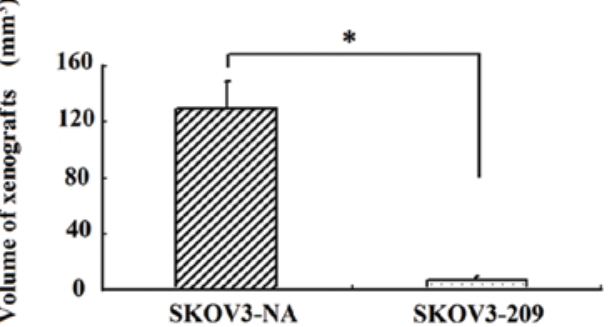

C
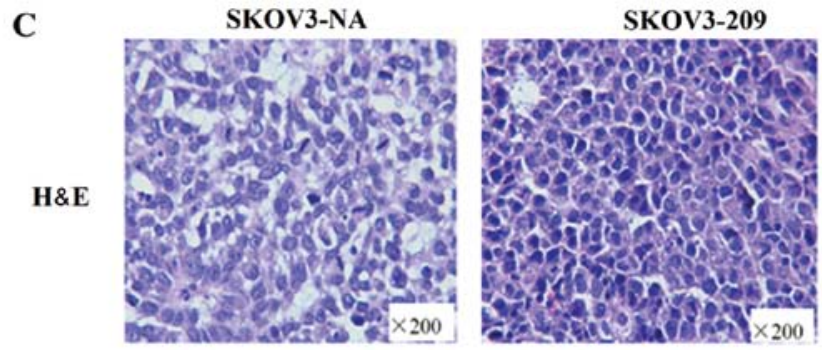

SKOV3-209

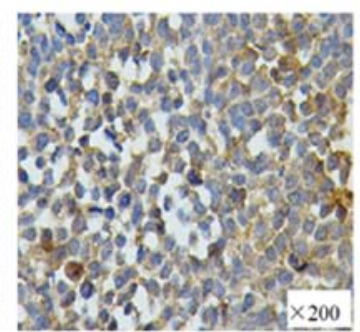

D

WFDC2
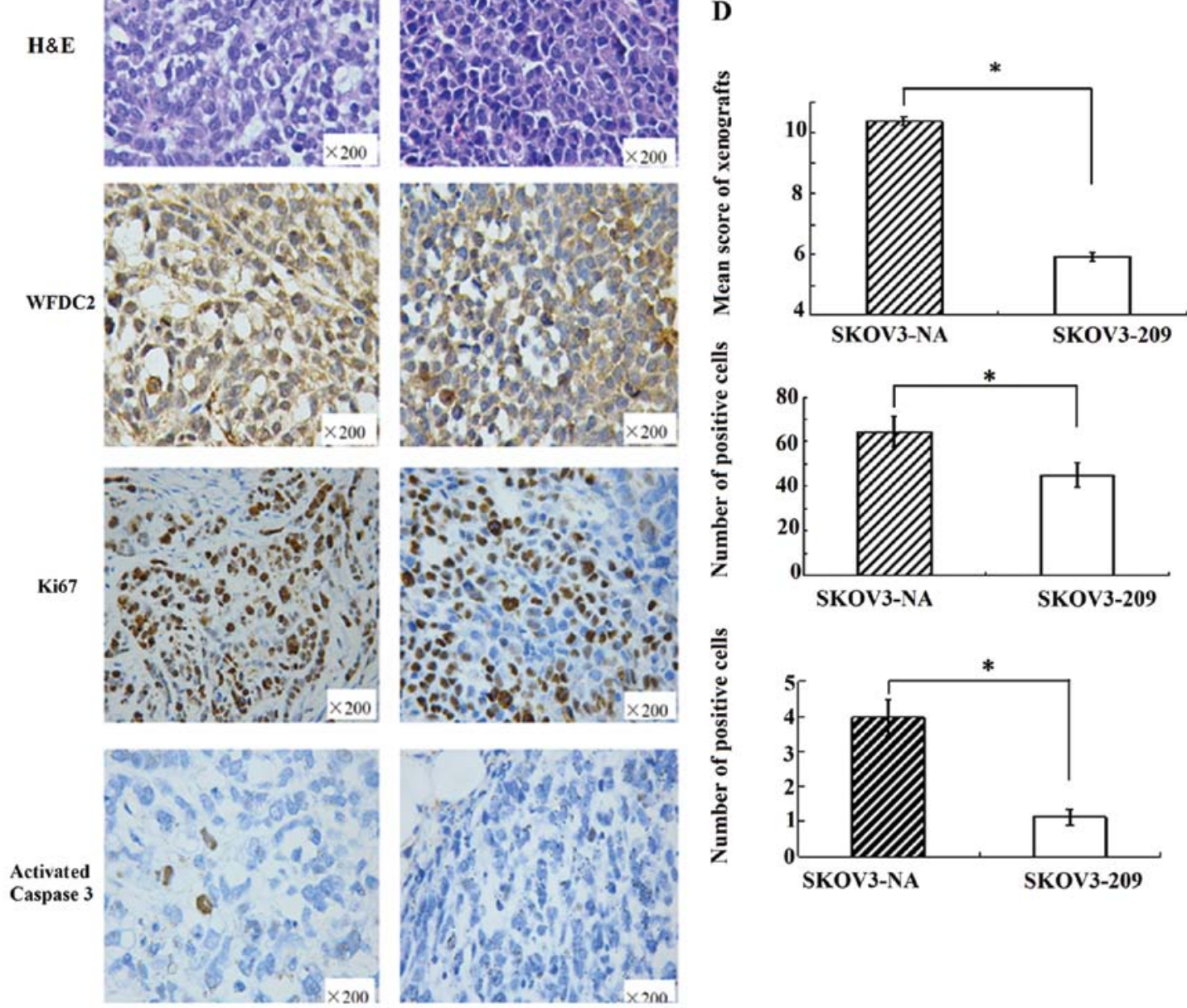

Figure 5. WFDC2 is required for SKOV3 cell tumorigenesis. (A) Mean tumor growth curves for SKOV3-209 and SKOV3-NA xenografts. "P<0.05 compared to SKOV3-NA. (B) Mean tumor volumes for SKOV3-209 and SKOV3-NA xenografts at 6 weeks. (C) Immunohistochemistry for WFDC2, Ki67 and activated caspase-3 in SKOV3-209 and SKOV3-NA xenografts. (D) Normalized WFDC2 and Ki67 protein levels in SKOV3-209 and SKOV3-NA xenografts. "P<0.05 compared to SKOV3-NA.

In order to further study the role of WFDC2 in tumor growth, we studied the correlation between WFDC2 and a series of genes related to cell proliferation and survival. Noteworthy is the identification of cyclin D1 as a downstream target of WFDC2. The ability of WFDC2 to induce cyclin D1 expression occurred at the levels of transcription and its expression was highly specific. This is also evident from the cell cycle of SKOV3 cells; WFDC2 gene silencing arrested the cell cycle in the G0/G1 phase. Cyclin D1 belongs to the core cell cycle machinery and it is frequently overexpressed in human cancers $(13,24,25)$. The strong positive correlation between WFDC2 and cyclin D1 expression observed in our study suggests that WFDC2 may be linked to the development and progression of ovarian cancer by regulating the cell cycle of ovarian cancer cells. Noteworthy, when WFDC2 was downregulated, nuclear localization of the Ki67 was reduced significantly in the xenografts. Ki67 is a nuclear protein that is necessary for cellular proliferation. The fraction of Ki67- 
positive tumor cells is often correlated with the clinical course of cancer, which makes it an excellent marker to determine the growth fraction of a given cell population in tumorigenicity (26-29). The positive correlation between WFDC2 and Ki67 may indicate the positive role of WFDC2 in tumor growth. Based on the combination of in vivo and in vitro experimental results, WFDC2 possibly plays an active role as a facilitator through regulation of cyclin D1 and Ki67 expression in tumor cell proliferation.

We also studied and analyzed the relationship between WFDC2 and apoptosis-related genes. In WFDC2 knockdown cells, a series of apoptosis-related gene expression levels were altered $(30,31)$. Fasl is an important apoptosis-related membrane protein and enhanced Fasl expression at mRNA and protein level caused by WFDC2 knockdown cells indicates that SKOV3-209 may more easily undergo apoptosis. However, in the in vitro flow cytometry experiments, WFDC2 gene knockdown did not lead to changes in the apoptosis rate of SKOV3 cells. The activation of caspase-3 levels was lower in the SKOV3-209 cells compared to the control group as demonstrated from the results of western blot analysis. In vivo, the activation of caspase-3 was very weak in both the WFDC2-knockdown and the control groups; the expression level in the knockout group was observed to be slightly higher.

Since these experimental results are contradictory, the role of WFDC2 in apoptosis remains unclear. In our experiments, SKOV3 cell apoptosis rate compared to only $2-5 \%$, the expression of apoptosis-related indicators, Fasl and activated caspase-3, in SKOV3 cells was low; thus further analysis is required to determine the relationship of WFDC2 and tumor cell apoptosis in different cell lines and clinical specimens.

In summary, the generation and subsequent use of a WFDC2 'knockdown' model has allowed for the initial elucidation of the molecular mechanism behind WFDC2 overexpression and tumor progession. In this study, we speculated and validated the association between cancer and WFDC2 and analyzed the potential role of WFDC2 in tumor progression. Our data suggest that WFDC2 is a proliferation inducer both in vitro and in vivo. The positive correlation between the expression of WFDC2 with cyclin D1 and Ki67 and the negative correlation with FasL was demonstrated. The cellular and molecular evidence demonstrates the promoting effect of WFDC2 in human ovarian cancer cell growth and tumorigenicity. These results strongly suggest the possible involvement of WFDC2 expression in tumor progression.

\section{Acknowledgements}

This study was supported by grants from the National High Technology Research and Development Program of China (863 Program), no. 2006AA02A311.

\section{References}

1. Kobel M, Kalloger SE, Boyd N, et al: Ovarian carcinoma subtypes are different diseases: implications for biomarker studies. PLoS Med 5: e232, 2008.

2. Bingle L, Singleton $\mathrm{V}$ and Bingle CD: The putative ovarian tumour marker gene HE4 (WFDC2), is expressed in normal tissues and undergoes complex alternative splicing to yield multiple protein isoforms. Oncogene 21: 2768-2773, 2002.
3. Hellstrom I, Raycraft J, Hayden-Ledbetter M, et al: The HE4 (WFDC2) protein is a biomarker for ovarian carcinoma. Cancer Res 63: 3695-3700, 2003.

4. Peters DG, Kudla DM, Deloia JA, et al: Comparative gene expression analysis of ovarian carcinoma and normal ovarian epithelium by serial analysis of gene expression. Cancer Epidemiol Biomarkers Prev 14: 1717-1723, 2005.

5. Galgano MT, Hampton GM and Frierson HF Jr: Comprehensive analysis of HE4 expression in normal and malignant human tissues. Mod Pathol 19: 847-853, 2006.

6. Palmer C, Duan X, Hawley S, Scholler N, Thorpe JD, Sahota RA, Wong MQ, Wray A, Bergan LA, Drescher CW, McIntosh MW, Brown PO, Nelson BH and Urban N: Systematic evaluation of candidate blood markers for detecting ovarian cancer. PLoS One 3: e2633, 2008.

7. Bingle L, Cross SS, High AS, et al: WFDC2 (HE4): a potential role in the innate immunity of the oral cavity and respiratory tract and the development of adenocarcinomas of the lung. Respir Res 7: 61, 2006.

8. Devoogdt N, Hassanzadeh Ghassabeh G, Zhang J, Brys L, De Baetselier P and Revets H: Secretory leukocyte protease inhibitor promotes the tumorigenic and metastatic potential of cancer cells. Proc Natl Acad Sci USA 100: 5778-5782, 2003.

9. Clauss A, Lilja $\mathrm{H}$ and Lundwall A: A locus on human chromosome 20 contains several genes expressing protease inhibitor domains with homology to whey acidic protein. Biochem J 368: 233-242, 2002

10. Israeli O, Goldring-Aviram A, Rienstein S, Ben-Baruch G, Korach J, Goldman B and Friedman E: In silico chromosomal clustering of genes displaying altered expression patterns in ovarian cancer. Cancer Genet Cytogenet 160: 35-42, 2005.

11. Hough CD, Cho KR, Zonderman AB, Schwartz DR and Morin PJ: Coordinately up-regulated genes in ovarian cancer. Cancer Res 61: 3869-3876, 2001.

12. Devoogdt N, Revets H, Kindt A, Liu YQ, De Baetselier P and Ghassabeh GH: The tumor-promoting effect of TNF-alpha involves the induction of secretory leukocyte protease inhibitor. J Immunol 177: 8046-8052, 2006.

13. Simpkins FA, Devoogdt NM, Rasool N, et al: The alarm antiprotease, secretory leukocyte protease inhibitor, is a proliferation and survival factor for ovarian cancer cells. Carcinogenesis 29: 466-472, 2008.

14. Zhang D, Simmen RC, Michel FJ, Zhao G, Vale-Cruz D and Simmen FA: Secretory leukocyte protease inhibitor mediates proliferation of human endometrial epithelial cells by positive and negative regulation of growth-associated genes. J Biol Chem 277: 29999-30009, 2002.

15. Clauss A, Ng V, Liu J, et al: Overexpression of elafin in ovarian carcinoma is driven by genomic gains and activation of the nuclear factor kappaB pathway and is associated with poor overall survival. Neoplasia 12: 161-172, 2010.

16. Tremblay GM, Sallenave JM, Israél-Assayag E, Cormier Y and Gauldie J: Elafin/elastase-specific inhibitor in bronchoalveolar lavage of normal subjects and farmer's lung. Am J Respir Crit Care Med 154: 1092-1098, 1996.

17. Taggart CC, Greene CM, McElvaney NG and O'Neill S: Secretory leucoprotease inhibitor prevents lipopolysaccharide-induced IkappaBalpha degradation without affecting phosphorylation or ubiquitination. J Biol Chem 277: 33648-33653, 2002.

18. Butler MW, Robertson I, Greene CM, O'Neill SJ, Taggart CC and McElvaney NG: Elafin prevents lipopolysaccharide-induced AP-1 and NF-kappaB activation via an effect on the ubiquitinproteasome pathway. J Biol Chem 281: 34730-34735, 2006.

19. Drannik AG, Henrick BM and Rosenthal KL: War and peace between WAP and HIV: role of SLPI, trappin-2, elafin and ps20 in susceptibility to HIV infection. Biochem Soc Trans 39: 1427-1432, 2011.

20. Velarde MC, Parisek SI, Eason RR, Simmen FA and Simmen RC: The secretory leukocyte protease inhibitor gene is a target of epidermal growth factor receptor action in endometrial epithelial cells. J Endocrinol 184: 141-151, 2005.

21. Westin U, Nystrom M, Ljungcrantz I, Eriksson B and Ohlsson K: The presence of elafin, SLPI, IL1-RA and STNFalpha RI in head and neck squamous cell carcinomas and their relation to the degree of tumour differentiation. Mediators Inflamm 11: 7-12, 2002.

22. Scott A, Weldon S and Taggart CC: SLPI and elafin: multifunctional antiproteases of the WFDC family. Biochem Soc Trans 39: 1437-1440, 2011. 
23. Cordes C, Hasler R, Werner C, et al: The level of secretory leukocyte protease inhibitor is decreased in metastatic head and neck squamous cell carcinoma. Int J Oncol 39: 185-191, 2011.

24. Barbieri F, Cagnoli M, Ragni N, Pedulla F, Foglia G and Alama A: Expression of cyclin D1 correlates with malignancy in human ovarian tumours. Br J Cancer 75: 1263-1268, 1997.

25. Shigemasa K, Hu C, West CM, et al: p16 overexpression: a potential early indicator of transformation in ovarian carcinoma. J Soc Gynecol Investig 4: 95-102, 1997.

26. Frutuoso C, Silva MR, Amaral N, Martins I, De Oliveira C and De Oliveira HM: Prognosis value of p53, C-erB-2 and Ki67 proteins in ovarian carcinoma. Acta Med Port 14: 277-283, 2001 (In Portuguese).

27. Kobel M, Turbin D, Kalloger SE, Gao D, Huntsman DG and Gilks CB: Biomarker expression in pelvic high-grade serous carcinoma: comparison of ovarian and omental sites. Int J Gynecol Pathol 30: 366-371, 2011.
28. Aune G, Stunes AK, Tingulstad S, Salvesen O, Syversen U and Torp SH: The proliferation markers Ki-67/MIB-1, phosphohistone $\mathrm{H} 3$, and survivin may contribute in the identification of aggressive ovarian carcinomas. Int J Clin Exp Pathol 4: 444-453, 2011.

29. Wang S, Ma XY, Xia Y and Zhang LH: Expressions of Ki67, PCNA and mitotic index in ovarian epithelial tumors. Sichuan Da Xue Xue Bao Yi Xue Ban 41: 575-580, 2010 (In Chinese).

30. Ma XY, He FX, Wu SF, Lu YP and Ma D: Expression of survivin in ovarian epithelial carcinoma and its correlation with expression of Fas and FasL. Ai Zheng 23: 173-176, 2004 (In Chinese).

31. Fassl S, Leisser C, Huettenbrenner S, et al: Transferrin ensures survival of ovarian carcinoma cells when apoptosis is induced by TNFalpha, FasL, TRAIL, or Myc. Oncogene 22: 8343-8355, 2003. 